\title{
Cinquantenaire de Sarennes
}

\author{
The Sarennes Jubile \\ par Philippe Huet, \\ IGGREF
}

Au terme de ces deux Journées, il faut d'abord remercier les organisateurs et tous ceux de France ou de l'Arc Alpin qui sont venus. A travers eux, c'est le groupe discret, courageux et efficace des observateurs en montagne enneigée qu'il faut saluer. Un véritable hommage leur est dû, et au-delà de Sarennes, c'est à l'ensemble des collecteurs de données sur la montagne enneigée qu'il faut rendre justice. A ce titre, permettez-moi d'associer au cinquantenaire de Sarennes le quasi-centenaire de l'enquête permanente avalanche (EPA), et les générations de forestiers, de routiers, de producteurs d'énergie, de personnels de stations, de chercheurs qui, par tous les temps, dans les endroits les plus éloignés, année après année ont observé, mesuré, rapporté, avec des matériels frustes ou sophistiqués, mais toujours en payant de leur personne.

Vous me permettrez quelques considérations sur la fonction d'observation, préparées avec Benoît LESAFFRE, chef du SRAE (Service de la recherche et des affaires économiques au ministère de l'Aménagement du Territoire et de I'Environnement - MATE), Paul BARON, de la MISE (Mission d'inspection spécialisée de l'environnement), et mes collègues de la mission en cours commandée par le MATE et le MENRT sur les "observatoires de l'Environnement", qui doit être achevée à l'été 2000.

- l'observation est et doit rester une science de la nature,

- le cadre social dans lequel elle s'exerce a beaucoup évolué,

- le cadre administratif et institutionnel a vieilli et ne s'est pas adapté,

- la fonction de l'observation doit être reconnue et organisée.

\section{I - L'OBSERVATION DES PHÉNOMÈNES NATURELS COMME CELLE DU MAN- TEAU NEIGEUX OU DES GLACIERS EST UNE SCIENCE DE LA NATURE}

L'observateur est un homme de terrain, un expert, ce qu'il voit, enregistre, et restitue, ne se réduit pas à des chiffres. "D'instinct", par "métier", il intègre ces chiffres, ces données dans le milieu où il les a recueillis, et il sait ainsi leur don- ner leur vraie valeur, leur vrai sens. Il suffit d'avoir écouté plusieurs années ici les hommes de la CLPA (carte de localisation probable des avalanches), débattre de la valeur et de la limite d'usage des documents qu'ils établissent, pour en être convaincu. La modélisation, chacun le sait, ne vaut que par la valeur des paramètres qui la nourrissent et la confrontation de ses résultats à l'observation. B. Lesaffre dit volontiers que l'art de l'ingénieur a voulu devenir science de l'ingénieur en passant par la technique, et qu'il faut maintenant, en assimilant science et technique, revenir à l'art. L'observation en est un élément essentiel.

\section{II $\square$ LE CADRE SOCIAL DE L'OBSERVATION A CONSIDÉRABLEMENT ÉVOLUÉ}

Ma génération, celle des sexagénaires, a vécu ces transformations. J'emprunte pour l'essentiel cette analyse à P. Baron. - 11 y a 35 ans, "l'Etat faisait tout" ; dans mon métier de génie rural, en haute Ariège, nous jaugions les sources d'altitude, hiver comme été, nous faisions les projets d'adduction d'eau, pour les communes, trouvions les financements, établissions les délibérations des Conseils municipaux, conduisions les travaux et assurions la gestion des syndicats intercommunaux. C'était exaltant et parfois dangereux.

- Est venue la décentralisation il y a 20 ans, l'Etat s'est replié au fur et à mesure que les Collectivités s'organisaient. Et les administrations déléguant aux établissements publics se sont interrogées sur leur raison d'être, abandonnant parfois - souvent, disent les mauvais esprits - l'esprit pour la lettre. Un repositionnement général s'est imposé.

- Arrive, sans temps de pause, une troisième période, celle du citoyen, de l'associatif - qui souhaite le représenter (mais quid de l'élu ?), du débat public, du judiciaire aussi. C'est aussi le temps du transfrontière.

- Lors de la première étape, l'Etat observait ce qu'il voulait avec ses propres moyens. Rappelez-vous pour le secteur commercial "le Corps des instruments de mesure" que certains en boutade souhaitent recréer pour d'autres buts. Plus sérieusement, je vous renvoie au rapport parlementaire Y. DAUGE sur la métrologie liée aux inondations. 
- Lors de la seconde étape, la Collectivité locale et les professionnels se sont mis aussi à observer pour leurs propres besoins sans coordination très forte avec les réseaux de l'Etat. Le Chercheur - je n'oublie pas que nous sommes ici sur un Campus - , a pu parfois bien distinguer sa fonction "noble" de celle de l'observateur, "le manuel", sans toujours bien réaliser que ses résultats étaient indissociables d'une observation de terrain continue et soignée.

- Nous sommes aujourd'hui dans la troisième étape, celle du débat public, de l'appropriation par chacun et l'observation doit jouer un rôle central :

- à l'amont d'une décision à prendre, pour que chacun dispose de la même information sur le milieu et que tous parlent de la même chose,

- à l'aval, parce que l'observation permet de suivre, d'évaluer, par des indicateurs les résultats obtenus.

La fonction d'observateur encadre et accompagne donc le débat, la décision et l'évaluation.

\section{LE CADRE INSTITUTIONNEL ET RÉGLEMENTAIRE N'A PAS ENCORE TOTALEMENT SUIVI ET MÉRITE UN SÉRIEUX AGGIORNAMENTO}

L'observation de Sarennes et surtout du système glaciaire français continue à relever du volontariat de quelques passionnés, malgré le caractère international des enjeux, (réchauffement climatique) et les signaux d'alarme répétés. Je me souviens de l'évaluation des programmes de nivologie, il y a plus de 10 ans où la question était évoquée ; ce travail remarquable, mené par F. VALLA pour le Cemagref et la station de l'Alpe d'Huez, valorisait plus, à l'époque, les chercheurs que les collecteurs. Je sais que l'évaluation scientifique de décembre 99 de ces mêmes programmes a conduit aux mêmes questions. Je sais aussi que le programme Sourhy pour Sarennes a amélioré les choses. Mais ailleurs ? Le beau dossier "glaciers à risques", présenté par le Cemagref à la DPPR (Direction de la pollution et de la prévention des risques) en février 1999 reste à examiner.

- "I'Enquête permanente avalanche" de génération administrative en génération administrative est passée des services forestiers à l'ONF (Office national des forêts), utilisée par le Cemagref et le RTM (restauration des terrains en montagne), sans que le caractère de service public de cette mission essentielle soit affirmé et clairement identifié dans des textes récents.

- le Cemagref a bâti et gère la carte de localisation probable des avalanches (CLPA) depuis 30 ans, sans que son statut d'établissement public à caractère scientifique et technique (EPST) lui permette d'afficher clairement que la CLPA est un service public, au même titre que la carte géologique. Là encore les textes ont vieilli.

IV D LA FONCTION D'OBSERVATION, ESSENTIELLE DANS LA NOUVELLE DONNE SOCIALE, CAR GARANTE DES AVENIRS POSSIBLES ET DU CONTRAT SOCIAL, DOIT ÊTRE RECONNUE ET ORGANISÉE

- Elle doit être clairement identifiée dans les métiers ("les instruments de mesure, l'hydrographe, le géographe,...), dans le statut des établissements et dans les budgets.

- Elle doit être organisée de façon coordonnée, pour les besoins de la "recherche et les besoins opérationnels". Ils sont spécifiques mais ne peuvent s'ignorer sans gaspillage, de temps, d'énergie, d'argent ; l'exemple de l'observation des sols en cours de mise en place par l'INRA et le MATE est une piste. La glace, indicateur d'évolution climatique, doit être surveillée régulièrement, à la fois pour la recherche et l'économie. Inutile de le souligner à Grenoble et au Cemagref, l'un des pères des BVRE (bassins versants représentatifs expérimentaux).

- Observer et restituer est une fonction de service public : les statuts des établissements publics qui s'y impliquent, ou à tout le moins, les conventions qui les lient à leur tutelle doivent décrire ces fonctions dans leur dimension technique, dans leur dimension de responsabilité, dans leur condition de mise à disposition, y compris juridique, dans leur exigence de moyens.

- Car observer a un coût, et dans la durée, un coût durable (je me souviens des exigences de Daniel DUBAND, qui m'indignait à l'époque lorsqu'il me disait "une station année EDF coûte $12 \mathrm{kF}$ ". Il avait raison de me le dire, même si j'avais raison de ne pas vouloir les payer. Aucun budget ni aucune déontologie admise par tous sur les conditions d'accès aux données n'existaient alors.

- Enfin, rapprocher les méthodes et comparer les résultats sur le plan international est devenu une exigence. L'Agence européenne pour l'Environnement est là pour la promouvoir.

\section{$\mathrm{V} \square \mathrm{CONCLUSION}$}

En conclusion, il est temps de construire cette fonction d'observation pour les phénomènes naturels indicateurs d'évolution des milieux, générateurs de risques et supports d'économie. Cet anniversaire de Sarennes vient à point pour le souligner.

La mission d'inspection (la MISE) à laquelle j'appartiens a deux occasions de faire avancer les idées auprès des pouvoirs publics :

- la première dans le cadre du retour d'expérience des événements avalancheux de l'hiver dernier que nous menons avec la RTM et le Cemagref, et qui peut permettre de faire le point sur la chaîne EPA (Enquête permanente avalanche) CLPA (carte de localisation probable des avalanches) - PPR (Plan de prévention des risques) et l'organisation qui en résulte avec une dimension internationale.

- la seconde est plus ambitieuse : c'est celle de la mission "Recherche / Environnement", que j'ai citée, commandée à la fois par M. M. Allègre et Mme Voynet, sur les "observatoires de l'environnement". Elle est conduite par F. Barthelemy, I. G. Mines, A. Riquois, IGGREF, X. Martin, ICGREF, le professeur Schlich, Directeur honoraire de recherche au CNRS, avec l'appui de l'IFEN. J'y ai été associé à son départ, d'autres missions m'en ont éloigné momentanément. Mais nous devons travailler ensemble dans la durée pour que cette reconnaissance et cette organisation nécessaire de la fonction d'observation, que j'ai rapidement décrite, soit une exigence qui fasse son chemin. Cette Journée y contribue. 